\title{
Une loi «hybride» qui altère sérieusement la crédibilité de la psychologie
}

La loi sur les professions de la psychologie présentée en consultation est clairement une loi hybride. Après avoir largement consulté sa base, la FMH ne soutiendra pas la LPsy sous cette forme là. En effet, elle mélange et juxtapose de manière inopportune la protection des titres dans le domaine de la psychologie avec la réglementation de l'une de ses pratiques: la psychothérapie non médicale ou psychologique. Or, nous savons tous que parmi les activités professionnelles de la psychologie, seul un petit nombre concerne la santé, les autres étant, par exemple, la psychologie de la circulation, la psychologie scolaire, la psychologie du travail, la psychologie génétique, la psychologie sociale ou la psychologie de l'apprentissage.

A l'origine pourtant, le projet se fondait sur un problème bien réel: la sécurité. Les motions Wicki et Triponez (2001) montraient alors avec évidence la nécessité d'une réglementation en ce qui concerne la protection des titres, l'assurance de la qualité et la protection des consommateurs dans le champ des professions de la psychologie. La nouvelle réglementation devait aussi éviter aux professionnels suisses de la psychologie une discrimination sur le marché européen.

Mais voilà, l'idée justifiée d'une protection globale a été prise en otage par une frange réduite de la psychologie (les psychothérapeutes), qui a opéré un intense travail de lobbying et de pressions à tous les niveaux. Sans la contestation et la résistance des facultés de psychologie, de la FMH et des sociétés de disciplines psychiatriques, c'est une loi sur la psychothérapie qui nous aurait été présentée. Les stigmates de cette tentative se retrouvent dans les expressions comme: «lutte contre les maladies», "garantir la protection de la santé» ou encore «établissement de diagnostics sûrs». Il est clair que l'enjeu de la loi n'est pas uniquement la protection des titres mais aussi l'occupation d'une place, injustifiée et indéfendable, dans le champ de la santé mentale et de la sécurité sociale.

Le corps médical n'a aucune raison ni aucune intention de s'opposer à des relations psychothérapiques de qualité entre les psychologues et leurs clients, surtout si elle vise l'exploration personnelle. Par contre, lorsqu'il s'agit de soigner spécifiquement une population qui souffre de maladies psychiques, nous sommes persuadés qu'une collaboration entre le corps médical et les psychologues formés à la psychothérapie est non seulement souhaitable mais aussi indispensable. J'en veux pour preuve la psychothérapie déléguée. Or, la LPsy qui nous est présentée n'encourage ni n'assure le maintien et développement de telles collaborations, mais elle induit pour le patient une confusion entre les professions médicales et psychologiques. De surcroît la psychologie s'y approprie l'exclusivité du nom de psychothérapeute!

Chaque année, 20 à $25 \%$ de la population est atteinte d'un trouble psychique clairement défini qui va au delà d'un «simple mal-être» (OMS, OBSAN). La prévention et les soins de la maladie mentale nécessitent donc la pose de diagnostics sûrs et d'indications précises quant au traitement à appliquer. Ces diagnostics et indications sont fondés sur des outils propres à la formation et à la pratique médicale. Ces actes médicaux s'appuient sur des études pré-/postgraduées et continues approfondies, sur la psychopathologie et la psychopharmacologie (cf. les traitements combinés), sur un examen clinique somatique minutieux, sur des examens complémentaires, ainsi que sur une approche bio-psycho-sociale à laquelle s'ajoute une formation psychothérapeutique spécifique. La formation en psychothérapie psychologique ne recouvre que partiellement ces champs de connaissance. C'est sans doute aussi la raison pour laquelle il a été refusé d'inclure les professions de la psychologie dans la loi sur les professions médicales (1997). La FMH est toujours convaincue que la responsabilité de la pose d'un diagnostic de maladie et de l'indication à un traitement spécifique est et doit rester médicale.

Vous l'aurez certainement compris, si la FMH soutient inconditionnellement le principe de la création d'une loi qui protège les titres de la psychologie, elle ne soutiendra pas cette version hybride de la LPsy. Son double statut ne fait que provoquer la confusion des compétences dans le champ des soins et tend surtout à substituer une profession de la santé par une autre discipline non médicale. Dans ces temps de «disette économique» la tentation de cette substitution est très forte, elle est même ouvertement évoquée par l'OFSP. Nous sommes convaincus que cette orientation se révélerait néfaste pour le patientconsommateur, dont il s'agit précisément d'assurer la protection dans un environnement social 
qui «consomme de plus en plus du psy» - avec le risque de diluer encore plus les diagnostics et les indications médicales dans des promesses de bonheur et dans un formatage social inapproprié.
Dans ce «marché du mental», la FMH plaide encore et toujours pour une collaboration interdisciplinaire constructive. Surtout dans le champ des psychothérapies.

Dr René Raggenbass, membre du Comité central de la FMH

\section{Ein hybrides Gesetz, das die Glaubwürdigkeit der Psychologie ernsthaft gefährdet}

Das Psychologieberufegesetz (PsyG), zu dem eine Vernehmlassung stattgefunden hat, ist ganz klar ein hybrides Gebilde. Nach breiter Konsultation der eigenen Basis ist die FMH zum Schluss gelangt, dass sie das PsyG in der vorliegenden Form nicht unterstützen wird. Der Entwurf vermischt und er stellt auf unzulässige Weise den Titelschutz in der Psychologie der Reglementierung einer ihrer praktischen Anwendungen gegenüber: der nichtmedizinischen oder psychologischen Psychotherapie. Nun, wir wissen alle, dass nur ein kleiner Teil der beruflichen Tätigkeiten in der Psychologie die Gesundheit betrifft. $\mathrm{Zu}$ den anderen Anwendungen gehören beispielsweise die Verkehrs-, die Schul- und die Arbeitspsychologie, die genetische Psychologie sowie die Sozial- und die Lernpsychologie.

Ursprünglich basierte der Entwurf allerdings auf einem tatsächlich existierenden Problem: der Sicherheit. Die Motionen Wicki und Triponez (2001) verwiesen damals klar auf den Regulierungsbedarf beim Titelschutz, bei der Qualitätssicherung und beim Konsumentenschutz auf dem Gebiet der psychologischen Berufe. Die neue Regelung sollte auch eine Diskriminierung der Schweizer Psychologen auf dem europäischen Markt vermeiden.

Das berechtigte Anliegen eines umfassenden Schutzes wurde jedoch von einer kleinen Minderheit der Psychologen - den Psychotherapeuten - in Beschlag genommen. Sie lobbyierten kräftig und übten auf allen Ebenen Druck aus. Ohne die Einwände und den Widerstand der psychologischen Fakultäten, der FMH und der psychiatrischen Fachgesellschaften hätte man uns ein Gesetz über die Psychotherapie vorgelegt. Die Spuren dieses Versuchs finden sich in Ausdrücken wie «Krankheitsbekämpfung», «Gesundheitsschutz» oder «Stellen von sicheren Diagno- sen». Es wird klar, dass es bei diesem Gesetz nicht nur um den Titelschutz geht, sondern darum, im Bereich der psychischen Gesundheit und der sozialen Sicherheit einen Platz zu besetzen, was ungerechtfertigt und nicht zu vertreten ist.

Die Ärzteschaft hat weder einen Grund noch die Absicht, sich gegen qualitativ hochstehende psychotherapeutische Beziehungen zwischen den Psychologen und ihren Klientinnen und Klienten auszusprechen, vor allem wenn damit die Erforschung der eigenen Persönlichkeit bezweckt wird. Wenn jedoch gezielt Menschen mit psychischen Erkrankungen behandelt werden sollen, ist nach unserer Überzeugung eine Zusammenarbeit zwischen den Ärzten und den psychotherapeutisch ausgebildeten Psychologen nicht nur wünschbar, sondern unerlässlich. Beweis dafür ist die delegierte Psychotherapie. Mit dem nun vorliegenden PsyG wird diese $\mathrm{Zu}$ sammenarbeit aber weder gefördert noch aufrechterhalten oder ausgebaut. Stattdessen führt es bei den Patientinnen und Patienten zu einer Verwirrung zwischen medizinischen und psychologischen Berufen. Ausserdem eignet sich die Psychologie so die Ausschliesslichkeit der Bezeichnung «Psychotherapeut» an!

Jedes Jahr leiden 20-25\% der Bevölkerung unter einer eindeutig definierten, psychischen Störung, die klar über eine einfache «Befindlichkeitsstörung» hinausgeht (WHO und OBSAN). Die Vorbeugung und Behandlung psychischer Erkrankungen erfordern folglich sichere Diagnosen und genaue therapeutische Indikationen. Diese Diagnosen und Indikationen basieren auf Werkzeugen, die zur medizinischen Ausbildung und Praxis gehören. Solche medizinischen Handlungen stützen sich auf eine tiefgreifende Aus-, Weiter- und Fortbildung, die Psychopathologie und Psychopharmakologie (vgl. die kombi- 
nierten Behandlungen), eine gründliche somatisch-klinische Untersuchung sowie auf Zusatzabklärungen auf biopsychosozialem Ansatz, der durch eine spezifische psychotherapeutische Ausbildung ergänzt wurde. Die Ausbildung im Bereich der psychologischen Psychotherapie deckt diese Wissensfelder nur teilweise ab. Deshalb wurde wohl auch eine Aufnahme der psychologischen Berufe ins Medizinalberufegesetz (1997) abgelehnt. Die FMH ist weiterhin der Überzeugung, dass die Verantwortung für die Diagnose einer Erkrankung und die Indikation einer spezifischen Behandlung beim Arzt, der Ärztin liegt und bleiben muss.

Die FMH unterstützt zwar bedingungslos den Grundsatz, dass es zum Schutz der Berufstitel in der Psychologie ein Gesetz braucht. Sie kann jedoch diesem hybriden Entwurf des PsyG nicht zustimmen. Dessen Doppelstatus führt zwangsläufig zu einer Kompetenzvermischung bei der Behandlung und ersetzt einen Gesundheitsberuf durch eine nichtmedizinische Disziplin. In diesen wirtschaftlich schwierigen Zeiten ist eine solche Versuchung gross, und diese Möglichkeit wird vom BAG sogar offen ausgesprochen. Wir sind überzeugt, dass sich eine solche Ausrichtung negativ auf die Patientinnen/Patienten und Konsumentinnen/Konsumenten auswirken würde. Genau diese Menschen wollen wir aber in einem sozialen Umfeld schützen, in dem immer mehr Psycholeistungen konsumiert werden. So laufen wir Gefahr, dass Diagnosen und medizinische Indikationen noch mehr zu Heilsversprechen und $\mathrm{zu}$ einer unangemessenen sozialen Formatierung verwässert würden.

Die FMH plädiert auf dem «Psychomarkt» weiterhin für eine konstruktive fächerübergreifende Zusammenarbeit - auch und gerade auf dem Gebiet der Psychotherapie.

Dr. med. René Raggenbass, Mitglied des Zentralvorstandes der FMH 